\title{
Fiscalidade ao Serviço da Saúde Pública: A Experiência na Tributação das Bebidas Açucaradas em Portugal
}

\section{Using Pricing Policies to Promote Public Health: The Sugar Sweetened Beverages Taxation Experience in Portugal}

\author{
Francisco GOIANA-DA-SILVA $\square^{1,2}$, Alexandre Morais NUNES ${ }^{3,4}$, Marisa MIRALDO ${ }^{5}$, Alexandra BENTO ${ }^{6}, \mathrm{João} \mathrm{BREDA}^{7,8}$, \\ Fernando Ferreira ARAÚJO 9,10 \\ Acta Med Port 2018 Apr;31(4):191-195 - https://doi.org/10.20344/amp.10222
}

Palavras-chave: Adoçantes Calóricos; Bebidas; Edulcorantes; Portugal; Saúde Pública; Taxas/economia; Taxas/legislação e jurisprudência

Keywords: Beverages; Nutritive Sweeteners; Portugal; Public Health; Sweetening Agents; Taxes/economics; Taxes/legislation \& jurisprudence

\section{Alimentação e saúde: perfil e comportamentos ali- mentares de risco da população Portuguesa}

As doenças crónicas são responsáveis por $80 \%$ da mortalidade nos países europeus, sendo as afeções do apareIho circulatório as principais responsáveis pela morte prematura..$^{1,2}$ Portugal acompanha esta tendência europeia ${ }^{1,3}$ que tende a agravar-se, se não foram implementadas medidas de promoção da saúde e prevenção da doença.

De forma a recolher informação nacional sobre o consumo alimentar (ingestão nutricional e dimensões sobre segurança alimentar) e sobre a atividade física e a sua relação com determinantes em saúde, foi retomado o inquérito Alimentar Nacional e de Atividade Física (IAN-AF), ${ }^{4}$ realizado uma única vez, em 1980, pelo Centro de Estudos de Nutrição do Instituto Nacional de Saúde Dr. Ricardo Jorge, com a colaboração do então Ministério da Agricultura e Pescas.

O mais recente $\mathrm{IAN}^{4}$ foi desenvolvido por iniciativa de um consórcio que envolveu um total de nove instituições e que teve como promotor a Faculdade de Medicina da Universidade do Porto. Tendo como base amostral o Registo Nacional de Utentes do SNS e como população alvo os cidadãos residentes em Portugal, foi avaliada uma amostra de 6553 cidadãos, entre outubro de 2015 e setembro de 2016, com idades compreendidas entre os três meses e os 84 anos e representativa da população portuguesa.

Entre os vários resultados alcançados concluiu-se que:

- $17 \%$ da população portuguesa ingere pelo menos um refrigerante ou néctar (220 g ou mais) por dia. Essa prevalência ascende aos $40,6 \%$ no grupo dos adolescentes;
- Mais de $95 \%$ da população portuguesa apresenta um consumo médio de açúcares simples superior a $10 \%$ do valor energético total diário, limite recomendado pela Organização Mundial de Saúde (OMS);

- $52,7 \%$ não cumpre a recomendação da OMS de consumir mais de $400 \mathrm{~g} / \mathrm{dia}$ de produtos hortícolas ou fruta.

Segundo a literatura naciona $\left.\right|^{5}$ e internacional, ${ }^{6}$ os comportamentos ligados aos estilos de vida são fatores determinantes do padrão epidemiológico. Como exemplo, a obesidade é o resultado de insuficientes níveis de atividade física combinados com uma alimentação inadequada, ${ }^{7,8}$ caracterizada por um consumo excessivo de calorias em grande parte, provenientes de açúcar e gordura. Acresce ainda que indivíduos com excesso de peso têm maior risco de consumo excessivo de sódio e de ácidos gordos saturados. Na população portuguesa, estes consumos contribuem para a perda de $15,4 \%$ dos anos de vida saudável. ${ }^{1}$ Neste âmbito, através do segundo IAN-AF estimou-se que:

- 5,9 milhões de portugueses têm excesso de peso;

- 8 em cada 10 idosos apresenta excesso de peso;

- Os indivíduos menos escolarizados apresentam maior prevalência de excesso de peso e de obesidade abdominal;

- Apenas $41,8 \%$ dos cidadãos apresenta uma prática regular de atividade física, desportiva e/ou de lazer programada. ${ }^{3}$

Paralelamente aos efeitos na morbilidade e mortalidade prematura, as doenças crónicas têm impacto significativo nas economias nacionais, entre outros fatores, pela

\footnotetext{
1. Estudante Doutorando em Politicas de Saúde. Department of Surgery and Cancer. Imperial College Medical School. Londres. Reino Unido.

2. Assistente Convidado de Gestão e Liderança em Saúde. Faculdade de Ciências da Saúde. Universidade da Beira Interior. Covilhã. Portugal.

3. Professor Auxiliar Convidado. Unidade Curricular de Políticas de Saúde. Instituto Superior de Ciências Sociais e Políticas. Universidade de Lisboa. Lisboa. Portugal.

4. Investigador. Centro de Administração e Políticas Públicas. Instituto Superior de Ciências Sociais e Políticas. Universidade de Lisboa. Lisboa. Portugal.

5. Associate Professor. Health Economics Department. Imperial College London Business School. Healthcare Management Group. Londres. Reino Unido.

6. Bastonária. Ordem dos Nutricionistas. Lisboa. Portugal.

7. Programme Manager. Nutrition, Physical Activity and Obesity. World Health Organisation. Lisboa. Portugal.

8. Head of the World Health Organisation European Office for Prevention and Control of Noncommunicable Diseases. Moscow. Russian Federation.

9. Secretário de Estado Adjunto e da Saúde. XXI Governo Constitucional. Lisboa. Portugal.

10. Professor Auxiliar Convidado. Faculdade de Medicina. Universidade do Porto. Porto. Portugal.

$\triangle$ Autor correspondente: Francisco Goiana-da-Silva. franciscogoianasilva@gmail.com

Recebido: 10 de janeiro de 2018 - Aceite: 06 de março de 2018 | Copyright @ Ordem dos Médicos 2018
} 
diminuição da produtividade, aumento do absentismo laboral e dos encargos com a saúde. ${ }^{4,6,9}$

Os estilos de vida condicionam a saúde individual ao longo do percurso de vida merecendo, neste contexto, as faixas etárias mais jovens particular atenção. A prevalência de situações de excesso de peso infantil constitui-se enquanto indicador de desafios de saúde que as sociedades enfrentarão no futuro e que terão impacto na sustentabilidade dos sistemas de saúde. Neste âmbito, através do segundo estudo COSI $2016^{10}$ estimou-se que:

- $30,7 \%$ das crianças portuguesas apresentam excesso de peso;

- $11,7 \%$ das crianças portuguesas são obesas.

Neste contexto, e de acordo com o relatório Health at a Glance 2017,11 que apresenta os principais indicadores da OCDE, a prevalência de excesso de peso nas crianças de diferentes idades em Portugal é superior à média dos países da OCDE.

\section{Estratégia Integrada para a Promoção da Alimenta- ção Saudável: reformulação}

O XXI Governo Constitucional, no seu programa para a saúde, assumiu como fundamental a política de promoção de uma alimentação saudável. Neste âmbito, os Ministérios das Finanças, Administração Interna, Educação, Saúde, Economia, Agricultura, Florestas e Desenvolvimento Rural e Mar desenvolveram uma Estratégia Integrada para a Promoção da Alimentação Saudável (EIPAS), publicada através do Despacho $n^{\circ}$ 11418/2017, de 29 de dezembro. A EIPAS organiza-se de acordo com quatro eixos estratégicos definidos de acordo com as recomendações da Organização Mundial de Saúde (OMS) neste âmbito ${ }^{12}$ :

- Eixo 1: Modificar o meio ambiente onde as pessoas escolhem e compram alimentos através da modificação da disponibilidade de alimentos em certos espaços físicos e promoção da reformulação de determinadas categorias de alimentos;

- Eixo 2: Melhorar a qualidade e acessibilidade da informação disponível ao consumidor, de modo a informar e capacitar os cidadãos para escolhas alimentares saudáveis;

- Eixo 3: Promover e desenvolver a literacia e autonomia para o exercício de escolhas saudáveis pelo consumidor;

- Eixo 4: Promover a inovação e o empreendedorismo direcionado à área da promoção da alimentação saudável.

As medidas fiscais com vista à regulação do setor alimentar enquadram-se no âmbito do Eixo 1.

3. Imposto Especial sobre o Consumo (IEC) de Bebidas adicionadas de Açúcar ou Edulcorantes: âmbito

A OMS defende que o consumo excessivo de açúcar é um dos principais responsáveis pelos problemas de obesidade, diabetes e queda dentária. As bebidas açucaradas são uma das principais fontes de açúcar nas dietas e o seu consumo tem vindo a aumentar na maioria dos países, especialmente entre as crianças e adolescentes. ${ }^{13} \mathrm{~A}$ evidência mostra que a política de preços constitui uma ferramenta para a promoção da alimentação saudável.14,15

Diversos países na região europeia introduziram regimes de taxação de gamas alimentares e nutricionais, motivados por razões do foro da saúde pública. ${ }^{16}$ Nos casos em que existe evidência científica disponível, esta parece ser consistente com a teoria económica e com os estudos científicos existentes, verificando-se diversos exemplos de alterações nos padrões de compra e consumo associados a políticas de regulação de preços. Neste contexto, a monitorização e avaliação das políticas em curso na região Europeia são uma prioridade. ${ }^{16}$

Em Portugal, o Orçamento do Estado para 2017, aprovado pela Lei n. ${ }^{\circ} 42 / 2016$, de 28 de dezembro, criou um novo Imposto Especial sobre o Consumo (IEC) que incidiu sobre bebidas adicionadas de açúcar ou outros edulcorantes, tais como refrigerantes, bebidas energéticas, águas aromatizadas e ainda concentrados de bebidas, sob a forma de xarope ou pó, que se destinem à preparação de bebidas semelhantes às referidas.

Sendo a unidade tributável constituída pelo número de hectolitros de produto acabado, que corresponde ao resultado da diluição e adicionamento de outros produtos para preparação da mistura final, as taxas aplicáveis foram as seguintes:

- Bebidas cujo teor de açúcar seja inferior a 80 gramas por litro: $€ 8,22$ por hectolitro;

- Bebidas cujo teor de açúcar seja igual ou superior a 80 gramas por litro: $€ 16,46$ por hectolitro;

- Concentrados cuja taxa é aplicável à mistura final.

\section{IEC de Bebidas adicionadas de Açúcar ou Edulco-} rantes: entrada em vigor

O IEC de Bebidas adicionadas de Açúcar ou Edulcorantes produziu efeitos a partir de 1 de fevereiro de 2017 . Foram enquadradas na abrangência do IEC e contabilizadas como inventário, à data da entrada em vigor da referida lei, as bebidas produzidas, importadas ou adquiridas.

\section{Bebidas adicionadas de Açúcar ou Edulcorantes: mercado}

As marcas abrangidas pela Associação Portuguesa de Bebidas Refrescantes Não Alcoólicas (PROBEB) foram responsáveis, durante o ano de 2014, pela transação de um volume de 441 milhões de litros bebidas adicionadas de açúcar ou edulcorantes comercializados em Portugal. Durante o ano de 2015 este valor situou-se nos $461,5 \mathrm{mi}-$ Ihões de litros de bebidas adicionadas de açúcar ou edulcorantes. Já no ano de 2016 foram transacionados pelas entidades representadas pela PROBEB 479,8 milhões de litros de bebidas adicionadas de açúcar ou edulcorantes, representando uma quota de mercado de $67 \%$ de todas as bebidas adicionadas de açúcar ou refrigerantes comercializados em Portugal (Fig. 1).

Durante o ano de 2017, a mesma associação representou um volume de 456,1 milhões de litros de bebidas 


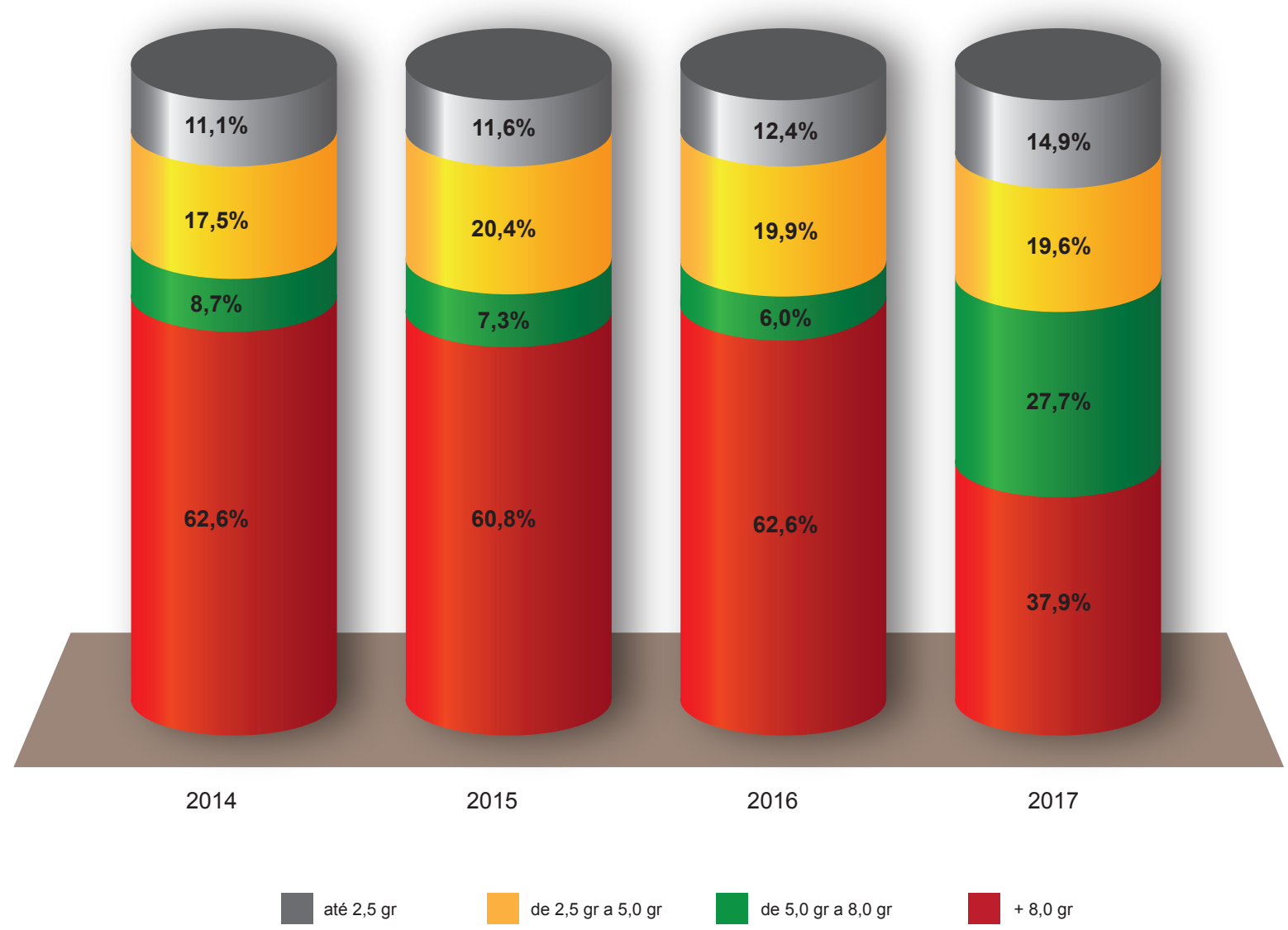

Figura 1 - Distribuição das bebidas com adição de açúcar consumidas pelos portugueses (quota de mercado PROBEB) - açúcar por 100 $\mathrm{ml}$ de bebida (em gramas). Fonte: PROBEB (2017).

adicionadas de açúcar no universo dos refrigerantes comercializados em Portugal, representando uma quota de mercado de $66 \%$. Durante o ano de 2017, verificou-se uma quebra de $4,3 \%$ de vendas relativamente ao período homólogo, não se tendo verificado alterações significativas da sua quota de mercado do tipo de bebidas abrangidas pelo IEC, o que pode significar que a redução do consumo foi transversal a todo o mercado.

\section{IEC: resultados preliminares}

Entre os anos de 2014 e 2016 verificou-se uma variação marginal entre os volumes relativos das gamas de bebidas com mais de 80 gramas de açúcar por litro e as restantes. Já durante o período de aplicação do IEC de bebidas adicionadas de açúcar ou edulcorantes, e de acordo com os dados da Autoridade Tributária e Aduaneira, verificou-se uma redução em quase $50 \%$ no consumo de bebidas enquadradas no escalão com mais de 80 gramas de açúcar por litro. Esta variação terá resultado de processos reformulação levados a cabo pela indústria ao nível das bebidas com maiores teores de açúcar adicionado. Verificou-se uma redução das quantidades de açúcar adicionado em determinados produtos que resultou na sua integração no grupo de bebidas com teor de açúcar inferior a 80 gramas por litro. Fatores como as alterações no padrão de escolha dos consumidores, em virtude do preço ou do marketing das empresas, não podem ser excluídos neste contexto, pelo que conclusões mais robustas implicarão um âmbito e período de análise mais alargados.

O fenómeno de transferência do consumo de bebidas com teores mais elevados de açúcar adicionado, incluídas no escalão superior de taxação, para o escalão com teores de açúcar inferiores teve como resultado uma diminuição de $15 \%$ do volume total de açúcar consumido através da gama de bebidas abrangidas pelo imposto. Este fenómeno representa, em termos nominais, um volume de 5630 toneladas de açúcar que os habituais consumidores deste tipo de bebidas ingeriram a menos durante o ano de 2017 (dados da PROBEB).

\section{Perspetivas futuras}

As doenças crónicas são, na sua maioria, de origem comportamental. Nesse contexto os hábitos alimentares desadequados assumem um papel relevante enquanto principal fator de risco evitável. De acordo com a literatura, ${ }^{17}$ as estratégias de alteração de hábitos alimentares por parte das populações devem ser transversais a diferentes áreas e abordagens, das quais se destacam a educação para a saúde, promoção da literacia, e as que visam alterações do ambiente alimentar e que podem ir desde a autorregulação (i.e. redução do sal no pão) a uma utilização inteligente da fiscalidade. No entanto, os regimes de tributação apresentam-se entre as medidas mais efetivas e mais céleres a obter efeitos. 
A aplicação de regimes de taxação de bebidas adicionadas de açúcar tem o potencial de reduzir os impacto das doenças provocadas pelo consumo excessivo de açúcar e dos custos a elas associados no curto prazo. ${ }^{18}$ Este facto é atualmente reconhecido por muitas entidades da saúde de grande reputação a nível mundial. ${ }^{19}$

Os resultados preliminares obtidos pela implementação do IEC de bebidas adicionadas de açúcar ou edulcorantes em Portugal parecem confirmar a hipótese referida na literatura, sendo que a redução no volume de açúcar ingerido através de bebidas em 2017 foi superior ao valor total de redução verificada, através de mecanismos de educação e autorregulação, entre os anos de 2013 e 2016.

A análise dos dados disponíveis evidencia, em 2017, uma enorme redução dos volumes tributados no escalão das bebidas com mais de 80 gramas de açúcar adicionado por litro, relativamente ao período homólogo anterior. Este fenómeno justifica-se pelo movimento de reformulação levado a cabo por algumas marcas do setor com vista a reduzir o teor de açúcar dos seus produtos, passando estes a ser tributados ao abrigo do escalão inferior. Não podem, neste contexto, ser ignorados eventuais impactos de quebras de consumo que poderão ter estado também associados às repercussões da implementação do IEC na reputação desta gama de bebidas. Também as transferências de consumo e substituições de consumo nos produtos mais açucarados, potenciados pela discussão pública alargada sobre o tema e pelo investimento preferencial no marketing dos produtos com menor teor de açúcar por parte da própria indústria constituem factores não negligenciáveis neste contexto. O estabelecimento de relações causais robustas neste âmbito implicará estudos adicionais para uma avaliação global.

O facto de a redução dos volumes incluídos no escalão superior de tributação ter sido mais marcada do que nas gamas com níveis de açúcar inferiores, aponta no sentido

\section{REFERÊNCIAS}

1. Global Burden of Disease Study 2015. Global Burden of Disease Study 2015 (GBD 2015) Results. Seattle, United States: Institute for Health Metrics and Evaluation (IHME); 2016. [consultado 2017 nov 09]. Disponível em: http://ghdx.healthdata.org/gbd-results-tool.

2. World Health Organization. The European health report 2012: charting the way to well-being. Copenhagen: WHO; 2013.

3. Direção-Geral da Saúde. Relatório do Programa Nacional para as Doenças Cérebro-Cardiovasculares. Lisboa: DGS; 2017.

4. IAN-AF - Inquérito Alimentar Nacional e de Atividade Física, 20152016. Apresentação sumária dos resultados; 2017 [consultado 2017 nov 09]. Disponível em: http://nutrimento.pt/activeapp/wp-content/ uploads/2017/03/IAN_Apresentacao-sumaria-resultados.pdf.

5. Comissão para os Determinantes Sociais da Saúde. Redução das desigualdades no período de uma geração. Igualdade na saúde através da acção sobre os seus determinantes sociais (Relatório Final). Lisboa: Organização Mundial da Saúde; 2010.

6. Withrow D, Alter D. The economic burden of obesity worldwide: a systematic review of the direct costs of obesity. Obes Rev. 2011;12:13141.

8. Organização para a Cooperação e Desenvolvimento Económico. Obesity and the economics of prevention: fit not fat. Paris: OECD Publishing; 2010.

9. Cecchini M, Sassi F, Lauer JA, Lee YY, Guajardo-Barron V, Chisholm D. Tackling of unhealthy diets, physical inactivity, and obesity: health effects and cost-effectiveness. Lancet. 2010;376:1775-84. de que a criação de escalões de tributação intermédios poderá alargar esse efeito. Dados os seus ganhos potenciais para a Saúde Pública, essa hipótese deveria ser avaliada no âmbito do Orçamento de Estado para 2019.

A análise do impacto de políticas de taxação, como o IEC português, na elasticidade dos preços dos produtos sobre os quais incidem é importante. Estudos internacionais estimam que a variação de preços pode ter influência na opção de substituição de alimentos pouco saudáveis por alimentos mais saudáveis. ${ }^{20}$ Assim, o desenvolvimento de estudos adicionais referentes ao IEC português reveste-se de grande pertinência, não apenas no contexto de uma eventual revisão da sua estrutura atual, mas também no âmbito internacional.

A maioria dos anos de vida saudável e recursos de saúde poupados graças a regimes de taxação semelhantes ao IEC em análise acumulam-se nos escalões socioeconómicos inferiores, isto é, trata-se de uma medida que combate as desigualdades em saúde. Ainda assim, com base numa análise aprofundada do impacto do IEC português nos diferentes escalões socioeconómicos, será essencial garantir a equidade desta política, nomeadamente através da aplicação dos recursos dela resultantes na promoção de uma alimentação mais saudável, especialmente nas classes mais carenciadas. $^{21}$

Os instrumentos fiscais, que incluem mas não se esgotam nos regimes de taxação como o IEC em análise, devem continuar a ser considerados e avaliados enquanto potenciais formas de abordagem de fatores de risco num contexto de saúde pública. ${ }^{22}$

\section{CONFLITO DE INTERESSES}

João Breda é funcionário da OMS. As suas declarações no ámbito deste trabalho científico são da sua exclusiva responsabilidade.

10. Bhattachary J, Sood N. Health insurance, obesity and its economic costs In: Philipson T, Dai C, Helmchen L, Variyam J, editors. The economics of obesity: a report on the workshop held at USDA's Economic Research Service. Washington: United States Department of Agriculture; 2004, p. 21-4.

11. Rito A, Cruz de Sousa R, Mendes S, Graça, P. Childhood Obesity Surveillance Initiative (COSI) Portugal 2016. Lisboa: Instituto Nacional de Saúde Doutor Ricardo Jorge; 2016.

12. Organização para a Cooperação e Desenvolvimento Económico. Health at a Glance 2017. Paris: OECD Publishing; 2017.

13. European food and nutrition action plan 2015-2020. Copenhagen: WHO Regional Office for Europe; 2014. [consultado 2018 Jan 09]. Disponível em: https://goo.gl/gozemR.

14. World Health Organization. Taxes on sugary drinks: Why do it? Copenhagen: WHO; 2016.

15. Woodward-Lopez G, Kao J, Ritchie L. To what extent have sweetened beverages contributed to the obesity epidemic? Public Health Nutr. 2010;14:499-509.

16. World Health Organization. Using price policies to promote healthier diets. Copenhagen: WHO; 2015.

17. Hyseni L, Atkinson M, Bromley H, Orton L, Lloyd-Williams F, McGill R, et al. The effects of policy actions to improve population dietary patterns and prevent diet-related non communicable diseases: scoping review. Eur J Clin Nutr. 2017;71:694-711.

18. Veerman JL, Sacks G, Antonopoulos N, Martin J. The impact of a tax 
on sugar-sweetened beverages on health and health care costs: a modelling study. PLoS One. 2016;11:e0151460

19. Backholer K, Martin J. Sugar-sweetened beverage tax: the inconvenient truths. Public Health Nutr. 2017;20:3225-7.

20. Andreyeva $T$, Long $M$, Brownell $K$. The impact of food prices on consumption: a systematic review of research on the price elasticity of demand for food. Am J Public Health. 2011;100:216-22.

21. Lal A, Mantilla-Herrera A, Veerman L, Backholer K, Sacks G, Siahpush
$\mathrm{M}$, et al. Modelled health benefits of a sugar-sweetened beverage tax across different socioeconomic groups in Australia: a cost-effectiveness and equity analysis. PLoS Med. 2017;14: e1002326.

22. Powell L, Chriqui J, Wada T, Chaloupka F. Assessing the potential effectiveness of food and beverage taxes and subsidies for improving public health: a systematic review of prices, demand and body weight outcomes. Public Health. 2012;14:110-28. 